\title{
ON PACKING MEASURES AND A THEOREM OF BESICOVITCH
}

\author{
IGNACIO GARCIA AND PABLO SHMERKIN
}

(Communicated by Tatiana Toro)

\begin{abstract}
Let $\mathcal{H}^{h}$ be the $h$-dimensional Hausdorff measure on $\mathbb{R}^{d}$. Besicovitch showed that if a set $E$ is null for $\mathcal{H}^{h}$, then it is null for $\mathcal{H}^{g}$, for some dimension $g$ smaller than $h$. We prove that this is not true for packing measures. Moreover, we consider the corresponding questions for sets of non- $\sigma$-finite packing measure and for pre-packing measure instead of packing measure.
\end{abstract}

\section{Introduction AND STATEMENTS OF REsults}

1.1. Introduction. We begin by recalling some definitions. A continuous nondecreasing function $h:(0, \infty) \rightarrow(0, \infty)$ that verifies $h(t) \rightarrow 0$ as $t \rightarrow 0$ is called a dimension function, and the set of these functions is denoted by $\mathcal{D}$.

We will be concerned with packing measures. Recall that, given $\delta>0$, a $\delta$ packing of a subset $E \subset \mathbb{R}^{d}$ is a collection of disjoint open balls centered at $E$ with diameter less than $\delta$. Given $h \in \mathcal{D}$, the $h$-dimensional packing pre-measure $P_{0}^{h}$ is given by

$$
\begin{aligned}
& P_{\delta}^{h}(E)=\sup \left\{\sum_{i} h\left(\left|B_{i}\right|\right):\left\{B_{i}\right\} \text { is a } \delta \text {-packing of } E\right\}, \\
& P_{0}^{h}(E)=\inf _{\delta>0} P_{\delta}^{h}(E)=\lim _{\delta \downarrow 0} P_{\delta}^{h}(E),
\end{aligned}
$$

where $|B|$ denotes the diameter of the ball $B$. The function $E \rightarrow P_{0}^{h}(E)$ is monotone and finitely subadditive, but fails to be countably subadditive, even on nice sets. The $h$-dimensional packing measure of $E$ (or $h$-packing measure of $E$ ) is defined by

$$
\mathcal{P}^{h}(E)=\inf \left\{\sum_{j=1}^{\infty} P_{0}^{h}\left(E_{j}\right): E \subset \bigcup_{j=1}^{\infty} E_{j}\right\}
$$

Received by the editors May 28, 2012 and, in revised form, July 24, 2012.

2010 Mathematics Subject Classification. Primary 28A78; Secondary 28A80.

Key words and phrases. Packing measure.

The first author was partially supported by CAI+D2009 No. 62-310 (Universidad Nacional del Litoral) and E449 (UNMDP).

The second author was partially supported by a Leverhulme Early Career Fellowship and by a Cesar Milstein Grant.

The authors thank the referee for helpful comments. 
It is well known that $\mathcal{P}^{h}$ is an outer measure on $\mathbb{R}^{d}$ (i.e. a monotone, countably subadditive set function which vanishes on the empty set), and is a measure (countably additive on disjoint collections) on the class of analytic (or Suslin) sets.

For a given dimension function $h$, the packing measure $\mathcal{P}^{h}$ is in some sense dual to the Hausdorff measure $\mathcal{H}^{h}$ (for whose definition the reader is referred to 4, Section 2.5]; in this note we do not use Hausdorff measures other than for motivation). Just as Hausdorff measures, packing measures are used to provide fine information on the size of fractal sets. For many random sets, especially related to Brownian motion, packing measures (rather than Hausdorff measures) provide the "right" concept to measure the size of the set; see e.g. [3] and [8] for two deep recent examples. Packing measures have also been recently applied to the study of Cantor sets defined in terms of their gaps [2] and their rearrangements [6].

Given $g, h \in \mathcal{D}$, we say that $g$ is a smaller dimension than $h$, denoted by $g \prec h$, whenever

$$
\lim _{t \rightarrow 0} \frac{h(t)}{g(t)}=0 .
$$

This relation defines a partial order on $\mathcal{D}$. A basic property of packing measures states that if $\left.\mathcal{P}^{h}\right|_{E}$ is a $\sigma$-finite nontrivial measure space, then $\mathcal{P}^{f}(E)=0$ for any $f \in \mathcal{D}$ such that $h \prec f$, and $\left.\mathcal{P}^{g}\right|_{E}$ is non- $\sigma$-finite for any $g \in \mathcal{D}$ such that $g \prec h$ (here $\left.\mathcal{P}^{h}\right|_{E}$ is the restriction of the measure $\mathcal{P}^{h}$ to the set $E$ ).

It follows from the above that the poset $\left\{f \in \mathcal{D}: \mathcal{P}^{f}(E)=0\right\}$ has no maximal elements, and a natural question is whether it may have minimal elements; likewise, one may ask whether $\left\{f \in \mathcal{D}:\left.\mathcal{P}^{f}\right|_{E}\right.$ is non- $\sigma$-finite $\}$ has maximal elements. In the context of Hausdorff measures, Besicovitch proved that the answer is always negative, at least if the set $E$ is analytic (see also [9, Theorem 42]). More precisely, we have

Theorem 1 ([1]). Let $E \subset \mathbb{R}^{d}$ and $h \in \mathcal{D}$.

(a) If $\mathcal{H}^{h}(E)=0$, then there exists $g \in \mathcal{D}$ such that $g \prec h$ and also $\mathcal{H}^{g}(E)=0$.

(b) If $E$ is analytic and $\left.\mathcal{H}^{h}\right|_{E}$ is non- $\sigma$-finite, then there exists $f \in \mathcal{D}$ such that $h \prec f$ and also $\left.\mathcal{H}^{f}\right|_{E}$ is non- $\sigma$-finite.

In this note we discuss Besicovitch's Theorem but in the setting of packing measures.

1.2. Results. Our first result is that the first part of Theorem 1 fails for packing measures. In fact, let $\mathcal{D}_{d} \subset \mathcal{D}$ be the set of the 'at most' $d$-dimensional doubling dimension functions, that is, functions $h \in \mathcal{D}$ that verify

$$
\frac{t^{d}}{h(t)}<c_{0}, \forall t>0, \quad \text { for some } c_{0}=c_{0}(h)
$$

and

$$
h(2 t) \leq c_{1} h(t), \forall t>0, \quad \text { for some } c_{1}=c_{1}(h) \leq 2^{d} .
$$

It is easy to see that, in fact, (2) implies the doubling condition (11). For example, $\mathcal{D}_{d}$ contains the power functions $t^{s}$ for $0<s \leq d$ as well as $t^{s} \varphi(t)$ when $\varphi(t)$ is slowly varying. 
Theorem 2. Given $h \in \mathcal{D}_{d}$, there is a Borel (in fact, $G_{\delta}$ ) set $E \subset \mathbb{R}^{d}$ such that $\mathcal{P}^{h}(E)=0$ but $\left.\mathcal{P}^{g}\right|_{E}$ is non- $\sigma$-finite for any $g \in \mathcal{D}$ such that

$$
\liminf _{t \rightarrow 0} \frac{h(t)}{g(t)}=0 .
$$

In particular, $\left.\mathcal{P}^{g}\right|_{E}$ is not $\sigma$-finite if $g \prec h$.

Now we turn to the second part of Besicovitch's Theorem. Recall that an analytic set is the continuous image of $\mathbb{N}^{\mathbb{N}}$, where $\mathbb{N}^{\mathbb{N}}$ is endowed with the product topology.

Theorem 3. Let $A \subset \mathbb{R}^{d}$ be an analytic set of non- $\sigma$-finite $\mathcal{P}^{h}$ measure. Then there exists $g \in \mathcal{D}$ such that $h \prec g$ and $A$ has non- $\sigma$-finite $\mathcal{P}^{g}$ measure.

Finally, for pre-packing measures we have the following general result.

Theorem 4. Let $A \subset \mathbb{R}^{d}$ and $h \in \mathcal{D}$.

(a) If $P_{0}^{h}(A)=0$, then there exists $g \in \mathcal{D}$ such that $g \prec h$ and $P_{0}^{g}(A)=0$.

(b) If $P_{0}^{h}(A)=+\infty$, then there exists $g \in \mathcal{D}$ such that $h \prec g$ and $P_{0}^{g}(A)=+\infty$.

In Section 2 we give the proofs of the results, and in Section 3 we conclude with remarks and a question.

\section{Proofs}

2.1. Proof of Theorem 2, In this note, $B_{r}(x)$ always denotes the open ball with center $x$ and radius $r$.

We need the following version of the mass distribution principle, which is an immediate consequence of the density theorem for packing measures (see [10] and also [7, Theorem 6.11] for the case when $h$ is a power function).

Lemma 5. Let $A \subset \mathbb{R}^{d}$ and $h \in \mathcal{D}_{d}$. If there is a finite Borel measure $\mu$ on $\mathbb{R}^{d}$ that verifies

$$
C^{-1} h(r) \leq \mu\left(B_{r}(x)\right) \leq C h(r), \forall r<1, \forall x \in A,
$$

for some positive and finite constant $C$, then $0<\mathcal{P}^{h}(A)<+\infty$.

Proof of Theorem 2. It is well known that a dense $G_{\delta}$ subset of $\mathbb{R}^{d}$ always has full packing dimension, even though it may have zero Lebesgue measure and even zero Hausdorff dimension. Our idea is to replace $\mathbb{R}^{d}$ by an appropriate set $K$ of positive, finite $h$-dimensional packing measure, construct such a dense $G_{\delta}$ set of zero $\mathcal{P}^{h}$ measure, and show that dense $G_{\delta}$ sets (relative to $K$ ) have non- $\sigma$-finite measure for any $g$ satisfying (3).

First step. Construction of the set $K$. Without loss of generality we assume that $c_{0}=1$, where $c_{0}$ is the constant in (1). Let $\left(a_{n}\right)_{n \geq 1}$ be defined by $h\left(a_{n}\right)=2^{-d n}$. From (11) and (2) we have that $2^{n} a_{n}<1$ and $2 a_{n+1}<a_{n}$, whence we can construct a $2^{d}$-corner set $K$ in the unit cube as follows. Let $K_{0}=[0,1]^{d}$ and define $K_{n}=$ $\bigcup_{j=1}^{2^{d}} Q_{j}^{n}$, for $n>0$, as the union of the $2^{d n}$ basic cubes $Q_{j}^{n}$ of side $a_{n}$ contained in $K_{n-1}$ that have a common vertex with a cube of $K_{n-1}$. Then $K=\bigcap_{n>0} K_{n}$.

Let $\mu$ be the uniform Cantor measure on $K$, which is constructed by repeated subdivision setting

$$
\mu\left(Q_{j}^{n}\right)=2^{-d n}, 1 \leq j \leq 2^{d n}, n \geq 0 .
$$


(See for example [4, Chapter 1].) Now fix $r<1$ and $x \in K$. Let $n$ be the greatest integer such that $B_{r}(x)$ contains a basic cube of $K_{n}$ but none of $K_{n-1}$. Then $a_{n} \leq 2 r$ and $r<\sqrt{d} a_{n-1}$, whence

$$
C h(r) \leq h\left(a_{n}\right) \leq c_{1} h(r),
$$

where $C>0$ is independent of $r$. Moreover, $B_{r}(x)$ intersects at most $2^{d+1}$ basic cubes of $K_{n}$. Then, by the definition of $a_{n}$, we have

$$
h\left(a_{n}\right) \leq \mu\left(B_{r}(x)\right) \leq 2^{d+1} h\left(a_{n}\right),
$$

and $0<\mathcal{P}^{h}(K)<+\infty$ by Lemma [5. Since $\mathcal{P}^{h}$ is invariant under isometries of $\mathbb{R}^{d}$, it assigns the same mass to all basic cubes of $K_{n}$ for all $n \geq 0$. Invoking also the Borel regularity of these measures, it follows that $\mu=\left.\left(1 / \mathcal{P}^{h}(K)\right) \mathcal{P}^{h}\right|_{K}$. In particular, $\mu$ and $\left.\mathcal{P}^{h}\right|_{K}$ have the same null sets.

Second step. Construction of the zero measure, dense $G_{\delta}$ set $E$. For $n \geq 0$, let $V_{n}$ be the set of all the vertices of basic cubes of $K_{n}$. Let

$$
U_{k}=\bigcup_{n \geq 0} \bigcup_{v \in V_{n}} K \cap B_{a_{2 n+k}}(v) \backslash\{v\} .
$$

Each $U_{k}$ is dense in $K$ and open with respect to the relative topology. We define $E=\bigcap_{k \geq 1} U_{k}$. The Baire category theorem implies that $E$ is a dense subset of $K$. Moreover, for each $k \geq 1$,

$$
\mu\left(U_{k}\right) \leq 2^{d+1} \sum_{n \geq 0} \sum_{v \in V_{n}} h\left(a_{2 n+k}\right)=\frac{2^{2 d+1}}{2^{d}-1} 2^{-d k},
$$

which implies $\mathcal{P}^{h}(E)=0$.

Third step. Conclusion of the proof. Now let $g \in \mathcal{D}$ satisfy (3). Let $\left(r_{k}\right)$ be a sequence decreasing to 0 such that $h\left(r_{k}\right) / g\left(r_{k}\right) \rightarrow 0$ and let $\left(n_{k}\right)$ be such that $a_{n_{k}+1}<r_{k} \leq a_{n_{k}}$.

We first claim that

$$
P_{0}^{g}(U \cap K)=+\infty \text { for any open set } U \subset \mathbb{R}^{d} \text {, with } U \cap K \neq \varnothing .
$$

Indeed, it is enough to show that $P_{0}^{g}(Q \cap K)=+\infty$ for each basic cube $Q$, but, since $P_{0}^{g}$ is invariant under Euclidian isometries, the symmetry of $K$ implies that $P_{0}^{g}(Q \cap K)$ is constant over all basic cubes in $K_{n}$ for each $n$ whence, by finite subadditivity of $P_{0}^{g}$, it is enough to show that $P_{0}^{g}(K)=+\infty$. Let $\delta>0$ and pick $k$ large enough that $r_{k}<\delta$. For each basic cube, let $v(Q)=\min \{v \in Q\}$, where the minimum is taken with respect to the lexicographical order (that is, $v(Q)$ is the down-left vertex of $Q)$. Then $\left\{B_{r_{k}}(v(Q)): Q\right.$ is a basic cube in $\left.K_{n_{k}-1}\right\}$ is a $\delta$-packing of $K$, and it follows that

$$
P_{\delta}^{g}(K) \geq 2^{d\left(n_{k}-1\right)} g\left(r_{k}\right)=\left(2^{d\left(n_{k}-1\right)} h\left(r_{k}\right)\right) \frac{g\left(r_{k}\right)}{h\left(r_{k}\right)} .
$$

Since $k$ is arbitrarily large, this shows that $P_{\delta}^{g}(K)=+\infty$ for all $\delta>0$, establishing (44).

Now suppose $E$ has $\sigma$-finite $\mathcal{P}^{g}$-measure. Then $E=\bigcup_{i} E_{i}$, where $\mathcal{P}^{g}\left(E_{i}\right)<+\infty$ for all $i$. In particular, each $E_{i}$ can be written as $E_{i}=\bigcup E_{i j}$, where $P_{0}^{g}\left(E_{i j}\right)<+\infty$ for all $i, j$. Since the packing pre-measure of a set and its closure are equal, we 
also have $P_{0}^{g}\left(\bar{E}_{i j}\right)<+\infty$ for all $i, j$. Hence, by claim (44), each $E_{i j}$ is nowhere dense. But this would imply that $K=(K \backslash E) \cup E$ is the union of two meager sets, contradicting the Baire category theorem. This contradiction finishes the proof.

2.2. Proof of Theorem 3. The proof of the theorem uses the ideas of Haase ([5], Theorem 2), where it is shown that an analytic set of non- $\sigma$-finite $h$-packing measure contains a compact subset of non- $\sigma$-finite $h$-packing measure. We provide full details for the reader's convenience.

We endow $\mathbb{N}^{\mathbb{N}}$ with the metric $\rho$ defined for $\mathbf{i}=\left(i_{1}, i_{2}, \ldots\right)$ and $\mathbf{j}=\left(j_{1}, j_{2}, \ldots\right)$ in $\mathbb{N}^{\mathbb{N}}$ by $\rho(\mathbf{i}, \mathbf{j})=1 / k_{0}$, where $k_{0}$ is the smallest integer $k$ such that $i_{k} \neq j_{k}$. This metric induces the product topology on $\mathbb{N}^{\mathbb{N}}$. Given $\left(i_{1}, \ldots, i_{k}\right) \in \mathbb{N}^{k}$, the cylinder in $\mathbb{N}^{\mathbb{N}}$ of level $k$ associated to $\left(i_{1}, \ldots, i_{k}\right)$ is the clopen set $G_{j_{1}, \ldots, j_{k}}=\left\{\mathbf{i} \in \mathbb{N}^{\mathbb{N}}: i_{1}=\right.$ $\left.j_{1}, \ldots, i_{k}=j_{k}\right\}$. For each $k \geq 1$, note that $\mathbb{N}^{\mathbb{N}}$ is the countable (disjoint) union of all the cylinders of level $k$. Also, each such cylinder has diameter $1 / k$.

We say that a set $E \subset \mathbb{R}^{d}$ has locally non- $\sigma$-finite h-packing measure if $U \cap E$ has non- $\sigma$-finite $h$-packing measure for each open set $U$ with $U \cap E \neq \emptyset$. We need a preliminary lemma. Let $\varphi: \mathbb{N}^{\mathbb{N}} \rightarrow A$ be a surjective continuous function, which exists because $A$ is analytic.

Lemma 6. Given $k>0$ and a closed set $C \subset \mathbb{N}^{\mathbb{N}}$ whose image $\varphi(C)$ has non- $\sigma$ finite h-packing measure, there is a closed subset $\tilde{C} \subset C$ such that $\varphi(\tilde{C})$ has locally non- $\sigma$-finite $h$-packing measure and $\operatorname{diam}(\tilde{C}) \leq 1 / k$.

Proof. Note that for some $\left(j_{1}, \ldots, j_{k}\right) \in \mathbb{N}^{k}$, the image of the closed set $D=$ $C \cap G_{j_{1}, \ldots, j_{k}}$ under $\varphi$ has non- $\sigma$-finite measure. Also, $D$ has diameter at most $1 / k$. Let

$$
\mathcal{U}=\{U: U \text { open, and } U \cap \varphi(D) \text { is nonempty and has } \sigma \text {-finite measure }\} .
$$

By the Lindelöf property, there is a countable subfamily $\left\{U_{n}\right\}$ of $\mathcal{U}$ for which $\bigcup_{n} U_{n}=\bigcup_{U \in \mathcal{U}} U$. Then $F=\varphi(D) \backslash \bigcup_{n} U_{n}$ has locally non- $\sigma$-finite measure. Hence, the set $\tilde{C}:=\varphi^{-1}(F) \cap D=D \backslash \varphi^{-1}\left(\bigcup_{n} U_{n}\right)$ verifies the statement of the lemma.

Proof of Theorem 3. We begin with some notation. For $\mathbf{i}=\left(i_{1}, i_{2}, \ldots\right) \in \mathbb{N}^{\mathbb{N}}$ let $\mathbf{i}_{n}=\left(i_{1}, \ldots, i_{n}\right)$. Also, $\mathbf{i}_{n} j$ denotes the $(n+1)$-tuple $\left(i_{1}, \ldots, i_{n}, j\right)$.

Let $C_{1} \subset \mathbb{N}^{\mathbb{N}}$ be the set obtained by applying Lemma $\left[6\right.$ to $C=\mathbb{N}^{\mathbb{N}}$ and $k=1$. Set $a_{2}=1$ and let $\left\{B_{r_{1 j}}\left(x_{1 j}\right)\right\}_{j \in \mathcal{I}}$ be a finite $a_{2}$-packing of $\varphi\left(C_{1}\right)$ such that

$$
\sum_{j \in \mathcal{I}} h\left(2 r_{1 j}\right)>4^{2}
$$

Since $\mathcal{I}$ is finite, there exists $0<a_{3}<\min _{j \in \mathcal{I}} 2 r_{1 j}$ such that if $y_{j} \in \bar{B}_{a_{3}}\left(x_{1 j}\right)$ for each $j \in \mathcal{I}$, then the balls $B_{r_{1 j}}\left(y_{j}\right)$ are disjoint. Next, for $j \in \mathcal{I}$, we define $C_{1 j} \subset C_{1}$ by applying Lemma 6 to $\varphi^{-1}\left(\bar{B}_{a_{3}}\left(x_{1 j}\right)\right) \cap C_{1}$ and $k=2$.

Continuing inductively in this fashion, we construct the following items:

(1) A sequence $\left(a_{n}\right)$ strictly decreasing to 0 .

(2) A subset $\mathcal{T} \subset \mathbb{N}^{\mathbb{N}}$ such that $\# \mathcal{I}(\mathbf{i}, n)<+\infty$ for each $\mathbf{i} \in \mathcal{T}$ and $n>0$, where

$$
\mathcal{I}(\mathbf{i}, n)=\left\{j \in \mathbb{N}: \mathbf{i}_{n} j=\mathbf{k} \text { for some } \mathbf{k} \in \mathcal{T}\right\}
$$


(3) A family of closed subsets $C_{\mathbf{i}_{n}} \in \mathbb{N}^{\mathbb{N}}$, indexed by taking $\mathbf{i} \in \mathcal{T}$ and $n>0$, that verifies
(a) $C_{\mathbf{i}_{n+1}} \subset C_{\mathbf{i}_{n}}$
(b) $\operatorname{diam} C_{\mathbf{i}_{n}} \leq 1 / n$;
(c) $\varphi\left(C_{\mathbf{i}_{n}}\right)$ has locally non- $\sigma$-finite $h$-packing measure.

(4) For each $\mathbf{i} \in \mathcal{T}$ and $n>0$, afinite $a_{n+1}$-packing $\left\{B_{r_{\mathbf{i}_{n} j}}\left(x_{\mathbf{i}_{n} j}\right)\right\}_{j \in \mathcal{I}(\mathbf{i}, n)}$ for the set $\varphi\left(C_{\mathbf{i}_{n}}\right)$ such that

(a)

$$
\sum_{j \in \mathcal{I}(\mathbf{i}, n)} h\left(2 r_{\mathbf{i}_{n} j}\right)>4^{n+1}
$$

(b) $a_{n+2}<2 r_{\mathbf{i}_{n} j}<a_{n+1}$ for all $j \in \mathcal{I}(\mathbf{i}, n)$;

(c) given $j \in \mathcal{I}(\mathbf{i}, n)$ and $y_{j} \in \bar{B}_{a_{n+2}}\left(x_{\mathbf{i}_{n} j}\right)$, the open balls $B_{r_{\mathbf{i}_{n} j}}\left(y_{j}\right)$ are pairwise disjoint;

(d) $\varphi\left(C_{\mathbf{i}_{n}}\right) \subset \bar{B}_{a_{n+1}}\left(x_{\mathbf{i}_{n}}\right)$.

The set $K=\bigcap_{n} \bigcup_{\mathbf{i} \in \mathcal{T}} C_{\mathbf{i}_{n}}$ is compact because it is closed and totally bounded (by (2) and (3)). Hence $E=\varphi(K)$ is a compact subset of $A$.

We define

$$
g(t)=\left\{\begin{array}{cc}
h(t) / 2^{n-\frac{t-a_{n}}{a_{n-1}-a_{n}}}, & t \in\left(a_{n}, a_{n-1}\right], n>1, \\
h(t), & t>a_{1} .
\end{array}\right.
$$

It is easy to check that $g$ is continuous and $h \prec g$.

Let $U$ be an open set such that $U \cap E \neq \emptyset$. Then there exist $\mathbf{i} \in \mathcal{T}$ and $n_{0}>0$ such that $\bar{B}_{a_{n+1}}\left(x_{\mathbf{i}_{n}}\right) \subset U$ for all $n \geq n_{0}$, whence $E \cap \bar{B}_{a_{n+1}}\left(x_{\mathbf{i}_{n}}\right) \neq \emptyset$ using the fact that $K \cap C_{\mathbf{i}_{n}} \neq \emptyset$ and (4)(d). In particular, for each $j \in \mathcal{I}(\mathbf{i}, n)$ there exists $y_{j} \in E \cap \bar{B}_{a_{n+2}}\left(x_{\mathbf{i}_{n} j}\right)$. By construction, the balls $B_{r_{\mathbf{i}_{n} j}}\left(y_{j}\right)$ are a packing of $E \cap U$, and by (4) (a) and (4) (b) we have

$$
P_{a_{n+1}}^{g}(U \cap E) \geq \sum_{j \in \mathcal{I}(\mathbf{i}, n)} g\left(2 r_{\mathbf{i}_{n} j}\right)>2^{n},
$$

whence $P_{0}^{g}(U \cap E)=+\infty$. By a Baire category argument (see [5, Lemma 4]), we conclude that the compact set $E$ has non- $\sigma$-finite $g$-packing measure, which implies that the same holds for $A$.

\subsection{Proof of Theorem 4.}

Proof of Theorem 4(国). It is enough to find $f \in \mathcal{D}$ such that $f \prec h$ and $P_{0}^{f}(A)<$ $+\infty$, because in this situation any $g \in \mathcal{D}$ such that $f \prec g \prec h$ satisfies the conclusion of the theorem.

Let $\left(\delta_{n}\right)_{n \geq 0} \searrow 0$ be a decreasing sequence of positive reals such that:

(1) $P_{\delta_{n}}^{h}(A) \leq 1 / 2^{2 n}$, and

(2) $2 h\left(\delta_{n+1}\right)<h\left(\delta_{n}\right)$.

If $0<\delta<\delta_{0}$ and $\mathcal{B}$ is a $\delta$-packing of $A$, set

$$
\mathcal{B}_{n}=\left\{B \in \mathcal{B}: \delta_{n+1} \leq|B|<\delta_{n}\right\} .
$$

Then, by (1),

$$
\sum_{n \geq 0} \sum_{B \in \mathcal{B}_{n}} 2^{n} h(|B|) \leq \sum_{n \geq 0} 2^{n} P_{\delta_{n}}^{h}(A) \leq \sum_{n \geq 0} 2^{-n}=2 .
$$


We define $f$ by

$$
f(t)=\left\{\begin{array}{cc}
\max \left(2^{n} h(t), 2^{n+1} h\left(\delta_{n+1}\right)\right), & t \in\left[\delta_{n+1}, \delta_{n}\right), \\
h(t), & t \geq \delta_{0} .
\end{array}\right.
$$

It follows from (2) that $f\left(\delta_{n}\right)=2^{n} h\left(\delta_{n}\right)$, hence $f$ is (left) continuous. It is also monotone nondecreasing and $f(t) \rightarrow 0$ as $t \rightarrow 0$ since

$$
f\left(\delta_{n}\right)=2^{n} h\left(\delta_{n}\right) \leq 2^{n} P_{\delta_{n}}^{h}(A) \leq 1 / 2^{n} .
$$

Moreover, $f \prec h$ because

$$
\frac{h(t)}{f(t)} \leq \frac{1}{2^{n}} \quad \text { for } t \in\left[\delta_{n+1}, \delta_{n}\right)
$$

Finally, for $t \in\left[\delta_{n+1}, \delta_{n}\right)$ we have $f(t) \leq 2^{n+1} h(t)$. Therefore if $\mathcal{B}$ is an arbitrary packing and $\mathcal{B}_{n}$ is as above, then

$$
\sum_{n \geq 0} \sum_{B \in \mathcal{B}_{n}} f(|B|)=\sum_{n \geq 0} \sum_{B \in \mathcal{B}_{n}} 2^{n+1} h(|B|) \leq 4,
$$

and the result follows.

For the proof of Theorem $4(\mathrm{~b})$ we use the following lemma.

Lemma 7. Let $C \subset \mathbb{R}^{d}$ and $h \in \mathcal{D}$. Then, $P_{0}^{h}(C)=+\infty$ if and only if there is a 1-packing $\left\{B_{j}\right\}$ of $C$ which verifies $\sum_{j} h\left(\left|B_{j}\right|\right)=+\infty$.

Proof. We assume that $C$ is bounded, for otherwise the equivalence is trivial from the definition of $P_{0}^{h}$.

Let $\delta>0$ and assume that there exists a 1-packing as in the statement. Then, there exists $J>0$ such that $\left|B_{j}\right|<\delta$ for all $j \geq J$, since $C$ is bounded. Hence $\left\{B_{j}\right\}_{j \geq J}$ is a $\delta$-packing and $\sum_{j \geq J} h\left(\left|B_{i}\right|\right)=+\infty$. Then, $P_{\delta}^{h}(C)=+\infty$ for all $\delta>0$, and therefore $P_{0}^{h}(C)=+\infty$.

Now suppose $P_{0}^{h}(C)=+\infty$. The packing is constructed as follows. We begin with a 1-packing $\left\{B_{i}^{1}\right\}_{i=1}^{N_{1}}$ of $C$ that satisfies:

- $\sum_{i=1}^{N_{1}} h\left(\left|B_{i}^{1}\right|\right)>2 h(1)$;

- $P_{0}^{h}\left(C \cap B_{N_{1}}^{1}\right)=+\infty$;

- $\operatorname{dist}\left(B_{i}^{1}, B_{N_{1}}^{1}\right)>0, \quad 1 \leq i<N_{1}$ (this holds because $h$ is left continuous).

As a consequence of these conditions, we can select a 1-packing $\left\{B_{i}^{2}\right\}_{i=1}^{N_{2}}$ of $C \cap B_{N_{1}}^{1}$ such that:

- $\sum_{i=1}^{N_{2}} h\left(\left|B_{i}^{2}\right|\right)>2 h(1)$;

- $P_{0}^{h}\left(C \cap B_{N_{2}}^{2}\right)=+\infty$;

- $\operatorname{dist}\left(B_{i}^{2}, B_{N_{2}}^{2}\right)>0$ and $\operatorname{dist}\left(B_{j}^{1}, B_{N_{2}}^{2}\right)>0, \quad 1 \leq i<N_{2}, 1 \leq j<N_{1}$;

- $B_{i}^{2} \cap B_{j}^{1}=\emptyset$, for all $1 \leq i \leq N_{2}, 1 \leq j<N_{1}$.

Then, $\left\{B_{1}^{1}, \ldots, B_{N_{1}-1}^{1}, B_{1}^{2}, \ldots, B_{N_{2}}^{2}\right\}$ is a 1-packing of $C$ of size

$$
\sum_{i=1}^{N_{1}-1} h\left(\left|B_{i}^{1}\right|\right)+\sum_{i=1}^{N_{2}} h\left(\left|B_{i}^{2}\right|\right)>3 h(1),
$$

where we used the fact that $\left|B_{N_{1}}^{1}\right|<1$ and the monotonicity of $h$. Continuing with this procedure ad infinitum we obtain the desired packing. 
Proof of Theorem 4(b). By Lemma 7, there is a 1-packing $\left\{B_{i}\right\}$ of $A$ such that

$$
\sum_{i} h\left(\left|B_{i}\right|\right)=+\infty
$$

Let $N_{1}, N_{2}, \ldots$ be an increasing sequence of integers such that

$$
\sum_{i \leq N_{j}} h\left(\left|B_{i}\right|\right)>4^{j} \quad \text { for all } j \geq 1 .
$$

Let $t_{j} \searrow 0$ be a sequence of reals such that $t_{j}<\min \left\{\left|B_{1}\right|, \ldots,\left|B_{N_{j}}\right|\right\}$ for all $j \geq 1$. We define $g:[0,+\infty) \rightarrow[0,+\infty)$ by

$$
g(t)=\left\{\begin{array}{cc}
2^{\frac{t-t_{j}}{t_{j-1}-t_{j}}-j} h(t), & t \in\left(t_{j}, t_{j-1}\right], j>1, \\
h(t), & t>t_{1} .
\end{array}\right.
$$

It is easily seen that $g \in \mathcal{D}$. Also, observe that for any $t \in\left(t_{j}, t_{j-1}\right]$,

$$
\frac{g(t)}{h(t)} \leq \frac{1}{2^{j-1}}
$$

whence $h \prec g$.

Now let $j>1$. Note that if $t_{j}<t$ for some $j$, then $t \in\left(t_{k}, t_{k-1}\right]$ for some $k \leq j$, and hence

$$
g(t) \geq \frac{h(t)}{2^{k}} \geq \frac{h(t)}{2^{j}} .
$$

(If $k=1$, then $t \in\left(t_{1},+\infty\right)$.) Therefore, we obtain

$$
\begin{aligned}
\sum_{i} g\left(\left|B_{i}\right|\right) & \geq \sum_{i: t_{j}<\left|B_{i}\right|} g\left(\left|B_{i}\right|\right) \\
& \geq \sum_{i: t_{j}<\left|B_{i}\right|} \frac{h\left(\left|B_{i}\right|\right)}{2^{j}} \\
& \geq \frac{1}{2^{j}} \sum_{i \leq N_{j}} h\left(\left|B_{i}\right|\right) \\
& >2^{j} .
\end{aligned}
$$

Hence $\left\{B_{i}\right\}$ is a 1-packing of $A$ such that $\sum_{i} g\left(\left|B_{i}\right|\right)=+\infty$, and the theorem follows by Proposition 7

\section{REMARKS AND A QUESTION}

We finish the article with some remarks and an open question.

Remark. In Theorem 2, we showed that for a large class of dimension fuctions $h$, there exist sets $E$ with the property that the poset $\left\{g \in \mathcal{D}: \mathcal{P}^{g}(E)=0\right\}$ has $h$ as a minimal element. We remark that, for general sets $E$, the poset may have no minimal elements. For example, if $K$ is the Cantor set constructed in the proof of Theorem 2 (for the given dimension function $h$ ), it is not hard to check that $\mathcal{P}^{g}(K)=0$ if and only if $h \prec g$, and this class clearly has no minimal elements (see [2] for the proof of the equivalence when $d=1$ ).

Remark. Theorem 3 can be generalized to a complete separable metric space, but by considering the radius-based definition of packing measure instead of the diameterbased definition (of course in $\mathbb{R}^{d}$ both definitions agree, but not in general metric spaces). Indeed, the method of Haase that we adapt works in that general setting. 
In Theorem 2, the sets we construct are $G_{\delta}$ but not closed. This suggests the following question:

Open question. Does there exist a closed set $E \subset \mathbb{R}^{d}$ such that the poset $\{h \in \mathcal{D}$ : $\left.\mathcal{P}^{h}(E)=0\right\}$ has a minimal element? If so, what are the possible minimal elements?

\section{REFERENCES}

[1] A. S. Besicovitch, On the definition of tangents to sets of infinite linear measure, Proc. Cambridge Philos. Soc. 52 (1956), 20-29. MR0074496 (17,595d)

[2] Carlos A. Cabrelli, Kathryn E. Hare, and Ursula M. Molter, Classifying Cantor sets by their fractal dimensions, Proc. Amer. Math. Soc. 138 (2010), no. 11, 3965-3974, DOI 10.1090/S0002-9939-2010-10396-9. MR2679618 (2011k:28011)

[3] Thomas Duquesne, The packing measure of the range of super-Brownian motion, Ann. Probab. 37 (2009), no. 6, 2431-2458, DOI 10.1214/09-AOP468. MR2573563 (2011b:28014)

[4] Kenneth Falconer, Fractal geometry, Mathematical foundations and applications, John Wiley \& Sons Ltd., Chichester, 1990. MR1102677 (92j:28008)

[5] H. Haase, Non- $\sigma$-finite sets for packing measure, Mathematika 33 (1986), no. 1, 129-136, DOI 10.1112/S0025579300013942. MR859505 (88a:28003)

[6] Kathryn Hare, Franklin Mendivil, and Leandro Zuberman. The sizes of rearrangements of cantor sets. Canad. Math. Bull. 56 (2013), no. 2, 354-365. MR3043062

[7] Pertti Mattila, Geometry of sets and measures in Euclidean spaces, Fractals and rectifiability, Cambridge Studies in Advanced Mathematics, vol. 44, Cambridge University Press, Cambridge, 1995. MR1333890 (96h:28006)

[8] Peter Mörters and Narn-Rueih Shieh, The exact packing measure of Brownian double points, Probab. Theory Related Fields 143 (2009), no. 1-2, 113-136, DOI 10.1007/s00440-007-0122-x. MR2449125(2009m:60192)

[9] C. A. Rogers, Hausdorff measures, Cambridge Mathematical Library, Cambridge University Press, Cambridge, 1998. Reprint of the 1970 original, with a foreword by K. J. Falconer. MR:1692618 (2000b:28009)

[10] S. James Taylor and Claude Tricot, Packing measure, and its evaluation for a Brownian path, Trans. Amer. Math. Soc. 288 (1985), no. 2, 679-699, DOI 10.2307/1999958. MR776398 (87a:28002)

Departamento de Matemática, Facultad de Ciencias Exactas y Naturales, Universidad Nacional de Mar del Plata, Mar del Plata, Buenos Aires Province, Argentina

E-mail address: nacholma@gmail.com

Department of Mathematics, Faculty of Engineering and Physical Sciences, UniverSity of Surrey, Guilford, GU2 7XH, United Kingdom

Current address: Torcuato Di Tella University, Av. Figeroa Alcorta 7350 (1428), Buenos Aires, Argentina

E-mail address: pshmerkin@utdt.edu 